\title{
Use of Alternative Reference Standards and Exempted Species To Evaluate the Performance of the Vitek 2 GP67 Cefoxitin/Oxacillin Screen for Coagulase-Negative Staphylococci
}

\author{
W. Michael Dunne, Jr., ${ }^{a}$ Rita Griffith, ${ }^{\text {b }}$ Virginia Shortridge ${ }^{\text {b }}$ \\ bioMérieux, Inc., Durham, North Carolina, USA a ; bioMérieux, Inc., St. Louis, Missouri, USA ${ }^{\text {b }}$
}

W e find it most interesting in the report of Johnson et al. (1) that Staphylococcus saprophyticus strains, which comprised the second most frequent representative of their collection of coagulase-negative staphylococci (CoNS), were included in all upfront calculations of the performance of the Vitek GP67 cefoxitin/ oxacillin screen for the detection of methicillin-resistant CoNS (see the abstract and Tables 1 and 2 in reference 1). This is despite the specific limitation taken by bioMérieux for this species with the cefoxitin screen test. Johnson et al. eventually provide corrected calculations excluding $S$. saprophyticus in the last paragraph of the results section, and indeed they note this limitation in Materials and Methods. From the standpoint of the reader, this presentation of data is somewhat misleading. Further, the statement in the initial paragraph of the discussion section stating that the Vitek cefoxitin screen does not meet FDA requirements is perplexing - especially considering that the reference standard required for the regulatory submission to the FDA was the cefoxitin disk test and not PCR for mecA. In fact, the submission data did meet FDA guidelines for performance using CoNS (201 challenge and clinical isolates, 11 species, three sites). From these data, it was recognized that this test tended to call more S. saprophyticus isolates resistant than did the cefoxitin disk reference method. Therefore, a limitation for S. saprophyticus was taken for the product, and customers were instructed to test all cefoxitin screen-positive S. saprophyticus strains with an alternate method before reporting. We appreciate that different strain collections from various geographic locations will likely generate variable results. However, it is difficult to draw comparisons from two disparate studies and conclude that the product does not meet FDA requirements, particularly when the comparison is to a different reference method.

\section{REFERENCE}

1. Johnson KN, Andreacchio K, Edelstein PH. 2014. Detection of methicillin-resistant coagulase-negative staphylococci by the Vitek 2 system. J Clin Microbiol 52:3196-3199. http://dx.doi.org/10.1128/JCM.01162-14.

Citation Dunne WM, Jr, Griffith R, Shortridge V. 2015. Use of alternative reference standards and exempted species to evaluate the performance of the Vitek 2 GP67 cefoxitin/oxacillin screen for coagulase-negative staphylococci. J Clin Microbiol 53:365. doi:10.1128/JCM.02771-14.

Editor: G. V. Doern

Address correspondence to W. Michael Dunne, Jr., william.dunne@biomerieux.co. For the author reply, see doi:10.1128/JCM.03023-14. Copyright @ 2015, American Society for Microbiology. All Rights Reserved. doi:10.1128/JCM.02771-14 